\title{
The impact of demographic changes, exogenous boosting and new vaccination policies on varicella and herpes zoster in Italy: a modelling and cost-effectiveness study
}

\author{
Alessia Melegaro ${ }^{1,2^{*}}$ (D), Valentina Marziano ${ }^{3}$, Emanuele Del Fava ${ }^{2}$, Piero Poletti ${ }^{3}$, Marcello Tirani ${ }^{4}$, Caterina Rizzo ${ }^{5}$ \\ and Stefano Merler $^{3}$
}

\begin{abstract}
Background: The present study aims to evaluate the cost-effectiveness of the newly introduced varicella and herpes zoster $(\mathrm{HZ})$ vaccination programmes in Italy. The appropriateness of the introduction of the varicella vaccine is highly debated because of concerns about the consequences on $\mathrm{HZ}$ epidemiology and the expected increase in the number of severe cases in case of suboptimal coverage levels.

Methods: We performed a cost-utility analysis based on a stochastic individual-based model that considers realistic demographic processes and two different underlying mechanisms of exogenous boosting (temporary and progressive immunity). Routine varicella vaccination is given with a two-dose schedule (15 months, 5-6 years). The $\mathrm{HZ}$ vaccine is offered to the elderly (65 years), either alone or in combination with an initial catch-up campaign (66-75 years). The main outcome measures are averted cases and deaths, costs per quality-adjusted life years gained, incremental costeffectiveness ratios, and net monetary benefits associated with the different vaccination policies.

Results: Demographic processes have contributed to shaping varicella and HZ epidemiology over the years, decreasing varicella circulation and increasing the incidence of $\mathrm{HZ}$. The recent introduction of varicella vaccination in Italy is expected to produce an enduring reduction in varicella incidence and, indirectly, a further increase of $\mathrm{HZ}$ incidence in the first decades, followed by a significant reduction in the long term. However, the concurrent introduction of routine $\mathrm{HZ}$ vaccination at 65 years of age is expected to mitigate this increase and, in the longer run, to reduce $\mathrm{HZ}$ burden to its minimum. From an economic perspective, all the considered policies are cost-effective, with the exception of varicella vaccination alone when considering a time horizon of 50 years. These results are robust to parameter uncertainties, to the two different hypotheses on the mechanism driving exogenous boosting, and to different demographic projection scenarios.

Conclusions: The recent introduction of a combined varicella and $\mathrm{HZ}$ vaccination programme in Italy will produce significant reductions in the burden of both diseases and is found to be a cost-effective policy. This programme will counterbalance the increasing trend of zoster incidence purely due to demographic processes.
\end{abstract}

Keywords: Varicella, Chickenpox, Herpes zoster, Shingles, Vaccination, Italy, Cost-effectiveness, Modelling, Individualbased models, Demography, Immunisation

\footnotetext{
* Correspondence: alessia.melegaro@unibocconi.it

${ }^{1}$ Department of Social and Political Sciences, Bocconi University, Milano, Italy

${ }^{2}$ Carlo F. Dondena Centre for Research on Social Dynamics and Public Policy,

Bocconi University, Milano, Italy

Full list of author information is available at the end of the article
}

(c) The Author(s). 2018 Open Access This article is distributed under the terms of the Creative Commons Attribution 4.0 International License (http://creativecommons.org/licenses/by/4.0/), which permits unrestricted use, distribution, and reproduction in any medium, provided you give appropriate credit to the original author(s) and the source, provide a link to the Creative Commons license, and indicate if changes were made. The Creative Commons Public Domain Dedication waiver (http://creativecommons.org/publicdomain/zero/1.0/) applies to the data made available in this article, unless otherwise stated. 


\section{Background}

Varicella zoster virus (VZV) is a DNA virus belonging to the Herpesviridae family that affects only humans. Infection by VZV can result in two distinct diseases: varicella or chickenpox, which is a highly communicable and widespread childhood disease, and herpes zoster (HZ) or shingles, caused by the reactivation of VZV, which remains latent in the dorsal root ganglia after primary varicella infection. Although it is usually a mild disease with a relatively low percentage of complications, especially when occurring in immunocompetent children, varicella is highly contagious and may lead to more severe consequences and disabling symptoms in adults. Reactivation of the virus, usually occurring in the elderly or immunocompromised patients, leads to $\mathrm{HZ}$ infection. This is characterised by a vesicular eruption along the course of the nerve and is commonly associated with pain. Complications of $\mathrm{HZ}$ occur in up to $20 \%$ of the cases among those aged 50 or older, with post-herpetic neuralgia (PHN) being the most common, persistent, and intractable chronic sequela $[1,2]$.

A live attenuated vaccine against varicella was developed in 1974 and introduced in some countries starting from 1995 [3]. In Italy, eight regions (Apulia, Basilicata, Calabria, Sardinia, Sicily, Tuscany, Veneto, and Friuli-Venezia Giulia) have gradually introduced childhood varicella vaccine into their immunisation programmes starting in 2003, in children aged 13-15 months and 5-6 years [4]. Since 2017, a two-dose schedule has been introduced nationally for all newborns as one of the ten vaccines (hexavalent, plus measles, mumps, rubella, and varicella) that have become compulsory for school attendance [5]. Nonetheless, in many developed countries, the introduction of varicella vaccination into the national schedule still represents an ongoing open discussion. Indeed, VZV mass immunisation would reduce varicella circulation, but it may potentially increase the incidence of more severe varicella cases among adults [6] and reduce the partial protection against HZ provided by VZV re-exposure (called "exogenous boosting"), thus increasing $\mathrm{HZ}$ incidence [7]. Results from $\mathrm{HZ}$ surveillance programmes in countries that have introduced VZV mass immunisation do not provide univocal evidence. Some countries detected an increase in $\mathrm{HZ}$ incidence following mass immunisation, while others did not observe any effect on it $[8,9]$. Conversely, an increase in $\mathrm{HZ}$ incidence has been reported in the past decades across various countries before the introduction of varicella vaccination programmes [10]. This pattern appears consistent with results from modelling work that showed that past demographic changes and, in particular, the ageing of the population may have generated the remarkable growth of HZ incidence observed in Spain between 1997 and 2004, before the introduction of the varicella vaccination programme [11].
A live attenuated vaccine against $\mathrm{HZ}$ was licenced in 2006 [12, 13], and so far has been recommended in some countries, either in combination with the varicella vaccination (e.g. in the USA and recently in Italy) or alone (e.g. in France and the UK), making the evaluation of post-vaccination trends even more complex.

Previous transmission models of VZV infection suggested an increase in $\mathrm{HZ}$ incidence as a consequence of the reduction of exogenous boosting associated with varicella vaccination. However, the magnitude of this increase depends on modelling assumptions on the mechanism of VZV reactivation [14-19], whose biology has not yet been elucidated [20-25]. The cost-effectiveness analysis here is performed under two different assumptions regarding the mechanism of VZV reactivation. The first assumption, which has been widely adopted in past modelling approaches, hypothesises temporary complete immunity to $\mathrm{HZ}$ following re-exposure to VZV [16]. The second assumption relies instead on the explicit modelling of the development of a progressive partial immunity to $\mathrm{HZ}$ following each re-exposure to VZV, which better reflects the biological mechanisms driving the exogenous boosting [7]. So far, models including the latter mechanism have provided a better fit to the age-specific profile of $\mathrm{HZ}$ incidence in several countries [26], Italy included [18].

Also, the cost-effectiveness of varicella vaccination programmes was shown to be highly dependent on the assumptions about the boosting mechanism [27, 28]. Varicella vaccination appeared cost-effective when the model excluded the effect of boosting on the epidemiology of $\mathrm{HZ}$ $[27,29]$. Conversely, when the latter effect was included, the cost-effectiveness of the programme became questionable, due to detrimental effects of varicella vaccination on $\mathrm{HZ}$ in the short and the medium term [30, 31].

The aim of this work is to provide a thorough evaluation of the expected effectiveness and cost-effectiveness of the recently introduced Italian varicella and $\mathrm{HZ}$ vaccination programme. For this purpose, a stochastic individual-based model (IBM) will be used, developed considering the observed demographic processes (such as the decline of fertility). Alternative immunisation strategies will be considered, and the sensitivity of our results to the two different assumptions about the exogenous boosting will be assessed.

\section{Methods}

In this study, we use a stochastic IBM for VZV transmission and reactivation in Italy, informed with historical demographic data and available demographic projections $[32,33]$ and calibrated on the age-specific varicella serological profile and age-specific $\mathrm{HZ}$ incidence.

The proposed modelling approach is similar to that adopted to investigate historical epidemiological trends of measles across different countries and varicella in Spain [11, 34, 35]. 
The model is used to assess the economic impact of different varicella and $\mathrm{HZ}$ vaccination strategies on the future epidemiology of the two diseases through a cost-effectiveness analysis. Details of the demographic and epidemiological data used to parameterise the model are provided in Additional file 1: Figures S1 and S4.

We consider two epidemiological models, which differ in the assumption made to model the mechanism driving exogenous boosting and VZV reactivation [7], denoted respectively as progressive immunity (PI) and temporary immunity (TI) $[16,18]$. The structures of the corresponding models are shown in Additional file 1: Figures S2 and S3. In both models, maternal antibodies confer protection against varicella infection to newborn babies for 6 months on average, after which children become susceptible to natural VZV (i.e. wild-type) infection. Susceptible individuals are exposed to a time- and age-dependent force of infection. After recovery, varicella-infected individuals acquire lifelong immunity against varicella. The generation time of varicella infection is assumed to be 3 weeks on average. In model PI, after recovery from varicella, individuals become susceptible to HZ. The rate of VZV reactivation decreases with the number of re-exposures to VZV, while it increases with both the time elapsed since the last re-exposure and the individual's age [18]. In model TI, individuals who recover from varicella acquire temporary full protection against $\mathrm{HZ}$, and the VZV reactivation rate only depends on the individual's age [16]. In both models, HZ-susceptible individuals may either develop HZ, acquiring, after recovery, lifelong immunity to $\mathrm{HZ}$ disease, or they can be boosted through VZV re-exposure. In model PI, each boosting event progressively reduces the risk of VZV reactivation into $\mathrm{HZ}$, whereas in model TI, it provides a temporary complete protection against $\mathrm{HZ}$ development. In both models, we assume that only a fraction of contacts with VZV-infected individuals results in an effective boosting event [36]. Epidemiological parameters of both models' structures are provided in the Additional file 1: Table S1 and S2.

Varicella vaccine is administered in our models starting from the year 2017. Vaccinated individuals either develop lifelong protection against varicella or they undergo vaccine failure. In the latter case, they remain susceptible to VZV and may experience a milder varicella infection, called "breakthrough varicella". Although individuals infected with breakthrough varicella can transmit the virus, they are assumed to be half as contagious as natural varicella cases [37].

Under model PI, individuals who have recovered from breakthrough varicella become susceptible to $\mathrm{HZ}$, whereas under model $\mathrm{TI}$, they become temporarily immune against HZ. In both models, varicella vaccinated individuals can develop HZ, either after recovery from varicella breakthrough or directly from the vaccine strain, or can experience boosting. The VZV reactivation rate for varicella vaccinated individuals is lower than for those who experienced natural varicella [38]. Individuals successfully vaccinated against $\mathrm{HZ}$ acquire lifelong immunity to VZV reactivation, whereas those experiencing $\mathrm{HZ}$ vaccine failure remain susceptible to $\mathrm{HZ}$.

Five different vaccination scenarios are considered and compared in an incremental cost-effectiveness analysis. The case of no intervention is also explored to assess the expected evolution of varicella and $\mathrm{HZ}$ epidemiology as driven by the changing demography only, had vaccination not been introduced. Following the new Italian National Immunisation Plan (NIP) 2017-2019 [39], we implement a routine varicella vaccination programme with a two-dose schedule (first dose at 15 months of age, second dose at 5-6 years of age) and an $\mathrm{HZ}$ vaccination programme with the live attenuated vaccine, targeted at individuals who are 65 years old. The two policies are evaluated either as single strategies or in combination. In addition, we also evaluate the effects of a catch-up campaign with the $\mathrm{HZ}$ vaccine targeting 66- to 75 -year-old individuals. The resulting five programmes are the following: (1) routine varicella vaccination $\left(V_{R}\right)$, (2) routine $H Z$ vaccination $\left(H Z_{R}\right)$, (3) routine $\mathrm{HZ}$ vaccination with $\mathrm{HZ}$ catch-up campaign $\left(\mathrm{HZ}_{\mathrm{R}+\mathrm{CU}}\right)$, (4) routine varicella and $H Z$ vaccinations $\left(V_{R} H Z_{R}\right)$, and (5) routine varicella and $\mathrm{HZ}$ vaccinations with $\mathrm{HZ}$ catch-up campaign $\left(\mathrm{V}_{\mathrm{R}} \mathrm{HZ}_{\mathrm{R}+\mathrm{CU}}\right)$. Base case coverage levels for varicella and $\mathrm{HZ}$ vaccination are assumed to be equal to $80 \%$ and $60 \%$, respectively. The vaccine efficacy per dose is set to $80 \%$ for the varicella vaccine, which implies an efficacy of $96 \%$ after two doses [40], while it is set to $50 \%$ for one dose of $\mathrm{HZ}$ vaccine [13]. The efficacy of varicella vaccine only refers to the protection against VZV infection. This means that a larger efficacy for the varicella vaccine generates in our model a larger proportion of individuals not developing varicella.

Finally, demographic changes are simulated in the period 2015-2100 as informed by temporal variations of the crude birth and age-specific mortality rates provided by the United Nations in the 2015 World Population Prospects [38].

The calibration of the models was carried out using Monte Carlo Markov chain (MCMC) methods applied to the binomial likelihood of the VZV seroprevalence profile in 1996-1997 [41] and to the Poisson likelihood of the age-specific HZ incidence in 2004 (Additional file 1: Figure S4) [2]. We calculated 95\% prediction intervals (PIs) for the model-based estimates. More details are provided in Additional file 1 where the robustness of our results is assessed (Figures S5 and S6). Modelling and data analyses were conducted in $\mathrm{C}$ and $\mathrm{R}$. 


\section{Cost-effectiveness analysis}

Cost-effectiveness analysis is applied to the outcomes of the epidemiological model, and quality-adjusted life years (QALYs) gained are used to evaluate the impact of different policies in terms of reduction of disease burden. Varicella cases are differentiated between natural infection and breakthrough cases, as the latter are expected to incur lower QALY losses and generate lower costs (Additional file 1: Figure S11). For HZ, we distinguish between cases that develop post-herpetic neuralgia (PHN) and those that do not, as costs and benefits for the two conditions are expected to differ (Additional file 1: Figure S12). We consider both the direct costs of disease (general practitioner (GP) visits, treatment, and hospitalisation) and the costs of the vaccination programmes. We report on the effects of the different policies on varicella and $\mathrm{HZ}$, in terms of both burden of illness (averted cases and deaths, by disease) and economic and quality of life impact (QALY gained $\Delta E$, and net costs $\Delta C$ ).

Cost-effectiveness outcomes are produced under the taxpayer perspective and evaluated at three different time horizons ( $\mathrm{TH}=25,50,85$ years), assuming discount rates of either $3 \%$ or $0 \%$ per year for both future health benefits and costs. The $3 \%$ discount rate puts less weight on the cases predicted in the long term, while the $0 \%$ discount rate weighs cases at a greater distance in time as much as those closer to the origin.

We perform an incremental cost-effectiveness analysis to determine, for each model and time horizon, which policies are deemed cost-effective, using both the incremental cost-effectiveness ratio (ICER, computed as $\Delta C$ / $\Delta E$ ) and the net monetary benefit (NMB, computed as $t \Delta E-\Delta C$ ), where the threshold $t$ represents the opportunity cost of an additional QALY gained. We consider two possible cost-effectiveness (CE) thresholds $t$, one demand-based of 40,000 EUR [42], and one supply-based of 15,000 EUR [43]. The former threshold represents the dominating approach in all health care systems, including those in Italy [42], and it depends on how individuals value health compared to other types of consumptions. The latter, based on the estimated marginal productivity of the health care system, represents a direct measure of the health consequence of changes in the allocation of the available resources [43]. A sensitivity analysis is conducted to assess how cost-effectiveness analysis results change when assuming different values of the CE threshold $t$.

The base case analysis considers 1000 model realisations of varicella and $\mathrm{HZ}$ cases by age and over time, generated under the strategies under investigation. These are combined with the base case values of the economic and quality of life parameters (Tables 1 and 2). The robustness of model results to uncertainty in model parameters is assessed through a probabilistic sensitivity analysis (PSA) [44], where, using an empirical Bayesian approach, the prior distributions for the model parameters are either grounded on the respective base case values or are set by assuming little or no information about the parameters of interest (e.g. when any estimate of the variability of the parameter is not available) [45]. For evaluating the effects of the uncertainty around both epidemiological estimates and economic model parameters, we compute the NMB associated with 1000 different parameter sets, sample from their posterior distribution, and derive 95\% credible intervals (CIs) from their posterior distribution. The uncertainty around the choice of the optimal strategy (i.e. generating the highest $\mathrm{NMB}$ ), under both discount rates of $0 \%$ and $3 \%$, is represented with (1) box plots of the posterior distribution of the NMB and (2) net benefit charts showing how the median NMB changes for a variety of values of the $\mathrm{CE}$ threshold [46]. Finally, six additional scenarios under the PSA are evaluated to assess the sensitivity of our results to (1) two different coverage levels of varicella vaccination (70\%, 95\%), (2) two extreme assumptions on the role of exogenous boosting (assuming either low or high reduction of the HZ risk due to VZV re-exposure), and (3) two different demographic scenarios on the total fertility rate in the future (a lower and a higher crude birth rate).

\section{Results}

Under the no vaccination scenario, both model structures predict a stable overall incidence of varicella over time (Fig. 1a), as well as an increase in $\mathrm{HZ}$ rate, which will stabilise only after some decades (Fig. $1 \mathrm{~d}-\mathrm{f}$ and Fig. 2). This growth can be ascribed to two factors, i.e. the population ageing that acts equally in both models, and the delayed effect, stronger for model PI, of the decline in the fertility rate which occurred during the last century on the individual risk of $\mathrm{HZ}$ development. Indeed, the decline of fertility in the past reduced both varicella circulation and the frequency of VZV re-exposure. In particular, during the period 2017-2100, model TI forecasts a peak in the total HZ incidence of about $15.1 \%$ (95\% PI $8.1-22.3 \%$ ) with respect to 2017 and a stable incidence level in the long term that is $2.6 \%$ (95\% PI -5.2 to $10.9 \%$ ) higher than in 2017. On the other hand, model PI forecasts a peak in HZ incidence with respect to 2017 that amounts to $61.4 \%$ (95\% PI 44.9-77.1\%) and a stable incidence level in the long term that is $48.3 \%$ (95\% PI 33.4-62.8\%) higher than in 2017 (Fig. 2).

The recently introduced combined varicella and zoster vaccination strategy $\left(\mathrm{V}_{\mathrm{R}} \mathrm{HZ} \mathrm{Z}_{\mathrm{R}}\right)$, at baseline coverage levels of $80 \%$ and $60 \%$, respectively, is expected to produce a sudden and enduring reduction in varicella incidence as well as a significant increase in the average age at 
Table 1 Epidemiological and quality of life (QALY) parameters of the economic model. We report the base case values and the standard deviations, taken either from the literature or from administrative data, the shapes of the prior distribution, the $95 \% \mathrm{Cl}$ from the posterior distribution of the parameters, and the source of the base case values

\begin{tabular}{|c|c|c|c|c|c|}
\hline Parameter & Base case values & Standard deviations & Prior Distribution & 95\% Cl Posterior distribution & Source \\
\hline \multicolumn{6}{|l|}{ Epidemiological parameters } \\
\hline Proportion of $\mathrm{HZ}$ cases developing $\mathrm{PHN}^{\mathrm{c}}$ & 0.049 (by age) & 0.0023 (by age) & Beta & - & [2] \\
\hline $\begin{array}{l}\text { Hosp. rate for natural varicella } \\
\text { (NV) per model TI/PI (per } 1000 \text { cases) }\end{array}$ & $\begin{array}{l}2.35 / 2.36 \\
\text { (by age) }\end{array}$ & 0.61/0.62 (by age) & Beta & - & Estimate $^{a}$ \\
\hline Breakthrough varicella (BV) vs. NV hosp. rate & 0.25 & 0.05 & Beta & {$[0.16-0.35]$} & {$[58,59]$} \\
\hline Hosp. rate for HZ per model TI/PI (per 1000 cases) & $\begin{array}{l}13.60 / 13.12 \\
\text { (by age) }\end{array}$ & 4.49/4.63 (by age) & Beta & - & Estimate $^{a}$ \\
\hline $\begin{array}{l}\text { Hosp. rate for PHN per model TI/PI } \\
\text { (per } 1000 \text { cases) }\end{array}$ & $\begin{array}{l}41.55 / 40.77 \\
\text { (by age) }\end{array}$ & 9.53/10.39 (by age) & Beta & - & Estimate $^{a}$ \\
\hline Case fatality rate for NV (per 1000 hospitalised) & 4.01 (by age) & 2.98 (by age) & Beta & - & Estimate $^{\mathrm{b}}$ \\
\hline BV vs. NV case fatality rate & 0.005 & 0.0022 & Beta & {$[0.002-0.01]$} & {$[59,60]$} \\
\hline HZ-PHN case fatality rate (per 1000 hospitalised) & 12.70 (by age) & 5.43 (by age) & Beta & - & Estimate $^{\mathrm{b}}$ \\
\hline \multicolumn{6}{|l|}{ No. GP consultations per NV case } \\
\hline$<14$ years & 2 & 0.2 & Gamma & {$[1.63-2.43]$} & [59] \\
\hline$\geq 15$ years & 1 & 0.2 & Gamma & {$[0.64-1.43]$} & [59] \\
\hline No. GP consultations per BV case & 0.5 & 0.05 & Gamma & {$[0.41-0.60]$} & [59] \\
\hline \multicolumn{6}{|l|}{ Quality of life measures } \\
\hline $\begin{array}{l}\text { Overall weighted health state index } \\
\left(E Q-5 D_{\text {index }}\right)\end{array}$ & 0.84 (by age) & 0.21 (by age) & Beta & & [61] \\
\hline \multicolumn{6}{|l|}{ Weighted health state index varicella } \\
\hline$<14$ years & 0.81 & 0.031 & Beta & {$[0.76-0.86]$} & [31] \\
\hline$\geq 15$ years & 0.73 & 0.025 & Beta & {$[0.68-0.78]$} & [62] \\
\hline Prob. severe NV cases & 0.65 & 0.0063 & Beta & {$[0.64-0.66]$} & [63] \\
\hline Prob. severe BV cases & 0.25 & 0.011 & Beta & {$[0.23-0.27]$} & [63] \\
\hline $\begin{array}{l}\text { Reduction in QALY loss } \\
\text { Mild vs. severe varicella cases }\end{array}$ & 0.25 & 0.10 & Beta & {$[0.08-0.47]$} & [48] \\
\hline \multicolumn{6}{|l|}{ QALY loss HZ } \\
\hline 20 years & 0.022 & 0.0018 & Beta & {$[0.019-0.026]$} & [48] \\
\hline 40 years & 0.031 & 0.0030 & Beta & [0.026-0.037] & [48] \\
\hline 60 years & 0.064 & 0.0082 & Beta & [0.049-0.081] & [48] \\
\hline 80 years & 0.19 & 0.030 & Beta & {$[0.14-0.25]$} & [48] \\
\hline
\end{tabular}

${ }^{a}$ Average number of hospitalisations by age due to varicella, HZ, and PHN (Hospital Discharge Register, 2001-2012) divided by the predicted pre-vaccination incidence generated by the epidemiological model. PHN incidence is derived by multiplying the estimated $\mathrm{HZ}$ incidence by the probability of $\mathrm{HZ}$ cases developing PHN [2]

${ }^{b}$ Average number of deaths by age due to varicella (Italian National Health Institute, 2001-2012) and HZ (European Union detailed mortality database, 2001-2012) divided by the respective estimates of the hospitalisation rates

${ }^{\mathrm{C}} \mathrm{PHN}$ cases lasting at least 3 months

varicella infection (Fig. 1b). Moreover, although in the short and the medium term the current programme might provide an increase in $\mathrm{HZ}$ incidence compared to the pre-vaccination level (though still lower than under the no vaccination scenario), in the long term it is expected to reduce the burden of $\mathrm{HZ}$ disease in Italy to its minimum, with small differences between the two models (Fig. 1d-f and Fig. 2, Additional file 1: Figures S9 and S10). In particular, we expect a reduction of 70.7\% (95\% PI 34.7-91.5\%) in HZ incidence with respect to the no vaccination scenario under model PI, and of 68.6\% (95\% PI 61.8-74.1\%) under model TI (Fig. 1f), with an upwards shift in the average age at VZV reactivation into $\mathrm{HZ}$. Indeed, for both models, while the age group that is mostly affected by HZ in 2017 is 55-64 years (accounting on average for $21.1 \%$ and $20.3 \%$ of the total cases in models PI and TI, respectively), in the long term the models forecast that most cases would occur in the age group 75-84 under model PI (40\% on average) and in the age group $85-99$ under model TI (26.8\% on average). 
Table 2 Cost of disease and vaccination parameters of the economic model. We report the base case values and the standard deviations, taken either from the literature or from administrative data, the shapes of the prior distribution, the $95 \% \mathrm{Cl}$ from the posterior distribution of the parameters, and the source of the base case values

\begin{tabular}{|c|c|c|c|c|c|}
\hline Parameter & $\begin{array}{l}\text { Base case } \\
\text { values (EUR) }\end{array}$ & $\begin{array}{l}\text { Standard } \\
\text { deviation (EUR) }\end{array}$ & Prior Distribution & $\begin{array}{l}95 \% \mathrm{Cl} \\
\text { Posterior distribution }\end{array}$ & Source \\
\hline \multicolumn{6}{|l|}{ Cost of disease parameters } \\
\hline \multicolumn{6}{|l|}{ GP consultation cost for $\mathrm{NV}^{\mathrm{a}}$} \\
\hline$<14$ years & 29.07 & 2.05 & Gamma & {$[25.14-33.27]$} & {$[64]$} \\
\hline$\geq 15$ years & 19.00 & 1.25 & Gamma & [16.65-21.53] & {$[65]$} \\
\hline \multicolumn{6}{|l|}{$\mathrm{GP}$ treatment cost for $\mathrm{NV}^{\mathrm{a}}$} \\
\hline$<14$ years & 13.64 & 1.61 & Gamma & [10.72-16.96] & {$[66]$} \\
\hline$\geq 15$ years & 26.10 & 2.00 & Gamma & {$[22.35-30.14]$} & {$[65]$} \\
\hline \multicolumn{6}{|l|}{ Hospitalisation cost for NV } \\
\hline$<14$ years & 2683.25 & 1780.56 & Gamma & [411.80-7128.02] & $\begin{array}{l}\text { Hospital Discharge } \\
\text { Register (HDR) Lombardy }\end{array}$ \\
\hline$\geq 15$ years & 2720.29 & 2573.64 & Gamma & [348.73-9463.01] & HDR Lombardy \\
\hline $\begin{array}{l}\text { Outpatient cost for } \mathrm{HZ} \\
\text { (incl. Visit, treatment, and diagnostics) }^{\mathrm{a}}\end{array}$ & 144.03 & 114.48 & Gamma & [11.53-438.21] & {$[2]$} \\
\hline $\begin{array}{l}\text { Outpatient cost for PHN } \\
\text { (incl. Visit, treatment, and diagnostics) }^{a}\end{array}$ & 523.72 & 520.05 & Gamma & [13.95-1905.90] & [2] \\
\hline \multicolumn{6}{|l|}{ Hospitalisation cost for $\mathrm{HZ}$} \\
\hline$<49$ years & 2073.50 & 2260.35 & Gamma & [176.35-8238.92] & HDR Lombardy \\
\hline$\geq 50$ years & 2020.23 & 1332.60 & Gamma & [307.63-5313.47] & HDR Lombardy \\
\hline \multicolumn{6}{|l|}{ Hospitalisation cost for PHN } \\
\hline$<49$ years & 1500.74 & 1714.41 & Gamma & [78.93-6132.75] & HDR Lombardy \\
\hline$\geq 50$ years & 1927.25 & 1892.80 & Gamma & [75.02-7058.80] & \\
\hline \multicolumn{6}{|l|}{ Vaccination parameters } \\
\hline Cost per dose of varicella vaccination & 31.46 & & Fixed & - & Purchase price ${ }^{b}$ \\
\hline Cost per dose of $\mathrm{HZ}$ vaccination & 87.00 & & Fixed & - & Invitation for bid \\
\hline Admin. cost per dose of vaccination ${ }^{a}$ & 7.56 & & Fixed & - & [59] \\
\hline
\end{tabular}

${ }^{a}$ These costs are adjusted for the inflation at 2015, using the Italian Consumer Price Index

${ }^{\mathrm{b}}$ Purchase price of the vaccine for varicella per dose, paid by Lombardy Regional Health System

Nonetheless, the overall reduction in $\mathrm{HZ}$ incidence obtained with the combined programme $\left(\mathrm{V}_{\mathrm{R}} \mathrm{HZ} \mathrm{Z}_{\mathrm{R}}\right)$ implies a much lower cumulative number of $\mathrm{HZ}$ cases and HZ-related deaths than those expected with no vaccination, under any time horizon (Additional file 1: Tables S3 and S4, with $3 \%$ and $0 \%$ discount rates, respectively).

The effect of the introduction of a varicella vaccination policy alone $\left(\mathrm{V}_{\mathrm{R}}\right)$ on natural HZ incidence in the short and the medium term strongly depends on the model considered. In the first decades after introduction, no evident variation with respect to the no vaccination scenario is expected under model TI, whereas a $15 \%$ increase of $\mathrm{HZ}$ incidence is estimated under model PI (Fig. 2). In the long term, since varicella vaccination would reduce the replacement of the HZ-susceptible individuals generated by natural varicella, we would find that $\mathrm{HZ}$ incidence is less than half of that expected under no vaccination (Fig. 1f), with levels even lower than those in the pre-vaccination period (Fig. 2). Conversely, a routine $\mathrm{HZ}$ vaccination programme would mitigate the increase of $\mathrm{HZ}$ incidence both in the absence $\left(\mathrm{HZ}_{\mathrm{R}}\right)$ and in the presence of varicella vaccination $\left(\mathrm{V}_{R} H Z_{R}\right)$, in both the short and the medium term. However, the policy including only $\mathrm{HZ}$ vaccination would not affect the replacement of the HZ-susceptible individuals caused by varicella infection, and therefore result in the long term in a much higher level of $\mathrm{HZ}$ incidence than that achieved through policy $V_{R}$ (Fig. 1f). Indeed, according to model PI, in the long term, $\mathrm{V}_{\mathrm{R}}$ and $\mathrm{HZ}_{\mathrm{R}}$ would respectively lead to a $60.6 \%$ (95\% PI $12.6-88.2 \%$ ) and an $18.1 \%$ (95\% PI $15.9 \%-20.4 \%$ ) reduction in $\mathrm{HZ}$ incidence with respect to no vaccination (although the undiscounted cumulative number of zoster cases remains slightly higher under policy $V_{R}$ (Additional file 1: Table S4).

Under both models and time horizons (except for $\mathrm{TH}=$ 50 under the PI model) and assuming a discount rate of $3 \%$, we found the combined policy with varicella vaccination and $\mathrm{HZ}$ vaccination with catch-up $\left(\mathrm{V}_{\mathrm{R}} \mathrm{HZ} \mathrm{R}_{\mathrm{R}} \mathrm{CU}\right)$ to 


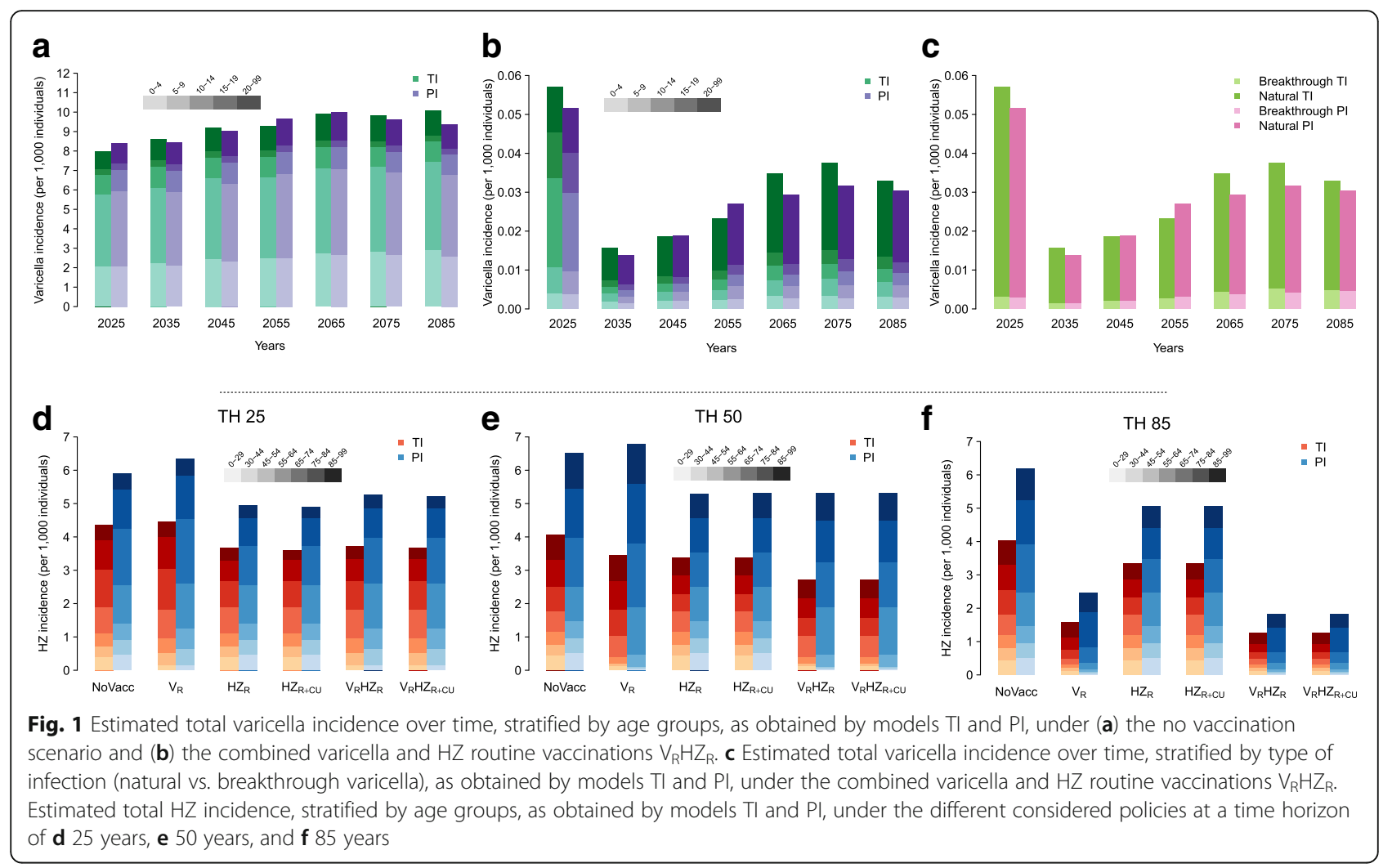

be the most cost-effective in terms of ICER and NMB, no matter what the chosen threshold (Table 3). However, for $\mathrm{TH}=50$ years and when considering model PI, the most cost-effective policy remains the one with the $\mathrm{HZ}$ vaccination and catch-up campaign $\left(\mathrm{HZ}_{\mathrm{R}+\mathrm{CU}}\right)$ (Table 3$)$.

Varicella vaccination alone $\left(V_{R}\right)$ was never found to perform better than the other strategies, even though it strongly dominated the no vaccination scenario under model TI, resulting in cost savings in the medium and in the long term (Table 3). Conversely, $\mathrm{V}_{\mathrm{R}}$ performed worse under model PI, where it turned out to be always dominated and even generated QALY losses in the medium and in the long term as a consequence of the increase in cumulative $\mathrm{HZ}$ cases in the first decades following the introduction of vaccination (Additional file 1: Table S3). Similar conclusions can be drawn when considering a discount rate of $0 \%$ (Additional file 1: Table S4). Also with undiscounted values, we found the combined policy

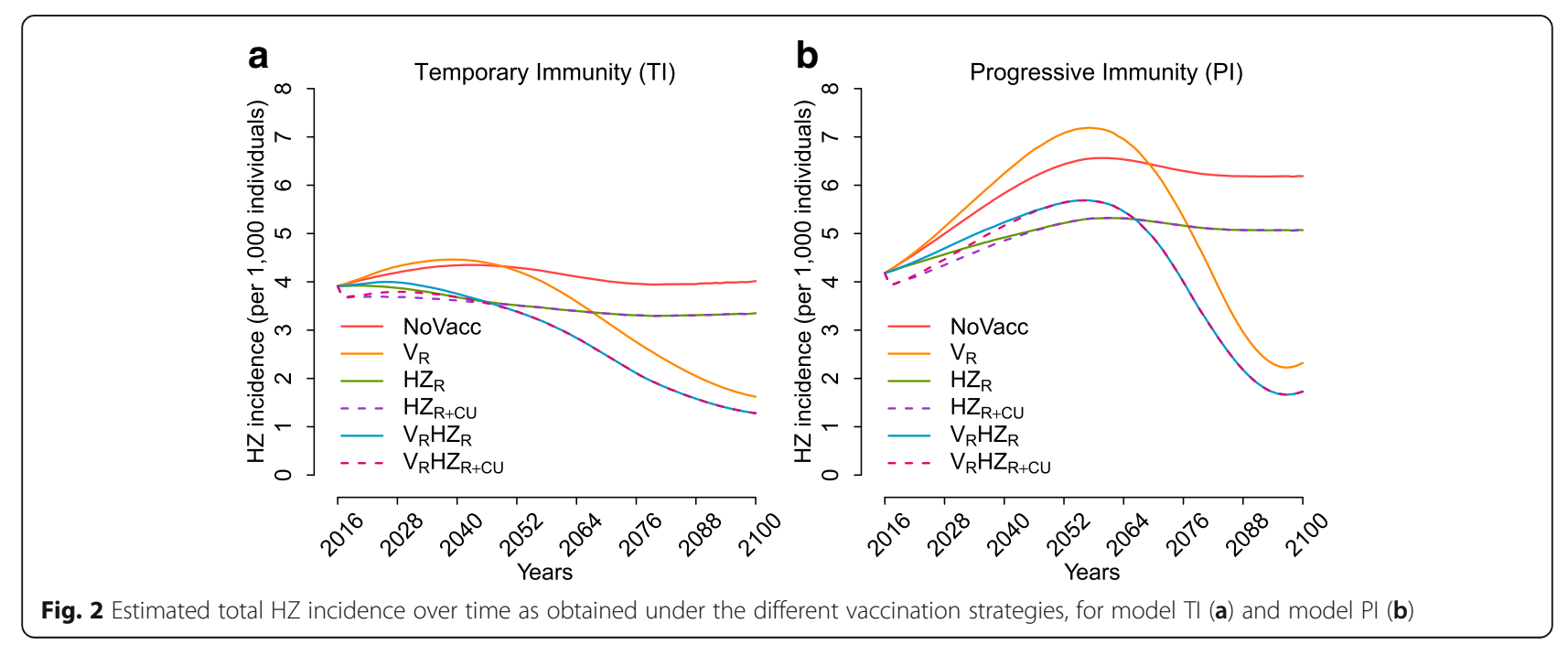


Table 3 Cost-utility analysis for the different vaccination policies, by model and by time horizon

\begin{tabular}{|c|c|c|c|c|c|c|c|c|c|c|c|}
\hline \multicolumn{6}{|l|}{ Model TI } & \multicolumn{6}{|l|}{ Model PI } \\
\hline Policy & Total cost ${ }^{a}$ & QALY loss ${ }^{b}$ & ICER $^{C}$ & $\mathrm{NMB}_{k}{ }^{\mathrm{d}}$ & $\mathrm{NMB}_{v}{ }^{\mathrm{e}}$ & Policy & Total cost ${ }^{a}$ & QALY loss ${ }^{\mathrm{b}}$ & ICER $^{C}$ & $\mathrm{NMB}_{\mathrm{k}}{ }^{\mathrm{d}}$ & $\mathrm{NMB}_{v}{ }^{e}$ \\
\hline \multicolumn{12}{|c|}{ Time horizon $=25$ years } \\
\hline No vacc. & 1812 & 455 & - & - & - & No vacc. & 2023 & 538 & - & - & - \\
\hline$V_{R}$ & 1831 & 446 & 2219 & 110 & 324 & $V_{R}$ & 2066 & 537 & WD & -37 & -28 \\
\hline$H Z_{R}$ & 2540 & 413 & WD & -94 & 963 & $H Z_{R}$ & 2722 & 481 & WD & 143 & 1548 \\
\hline$V_{R} H Z_{R}$ & 2555 & 402 & WD & 50 & 1371 & $V_{R} H Z_{R}$ & 2757 & 477 & WD & 166 & 1667 \\
\hline $\mathrm{HZ} \mathrm{R}_{\mathrm{R}+\mathrm{CU}}$ & 2823 & 380 & WD & 109 & 1975 & $\mathrm{HZ} \mathrm{R}_{\mathrm{R}} \mathrm{CU}$ & 2995 & 444 & WD & 436 & 2782 \\
\hline $\mathrm{V}_{R} \mathrm{HZ} \mathrm{Z}_{\mathrm{R}+\mathrm{CU}}$ & 2836 & 369 & 12,989 & 265 & 2414 & $\mathrm{~V}_{\mathrm{R}} \mathrm{HZ} \mathrm{Z}_{\mathrm{R}+\mathrm{CU}}$ & 3028 & 439 & 10,175 & 476 & 2944 \\
\hline \multicolumn{12}{|c|}{ Time horizon $=50$ years } \\
\hline No vacc. & 2668 & 691 & - & - & - & No vacc. & 3145 & 885 & - & - & - \\
\hline$V_{R}$ & 2643 & 680 & Cost-saving & 190 & 466 & $V_{R}$ & 3240 & 924 & SD & -679 & -1654 \\
\hline$V_{R} H Z_{R}$ & 3563 & 576 & WD & 820 & 3679 & $H Z_{R}$ & 3989 & 745 & 6031 & 1255 & 4754 \\
\hline$H Z_{R}$ & 3601 & 593 & SD & 531 & 2970 & $V_{R} H Z_{R}$ & 4047 & 767 & SD & 878 & 3844 \\
\hline $\mathrm{V}_{\mathrm{R}} \mathrm{HZ} \mathrm{R}_{\mathrm{R}+\mathrm{CU}}$ & 3841 & 543 & 8722 & 1052 & 4760 & $\mathrm{HZ} \mathrm{R}_{\mathrm{R}+\mathrm{CU}}$ & 4258 & 707 & 6984 & 1563 & 6022 \\
\hline$H Z_{R+C U}$ & 3880 & 560 & SD & 749 & 4017 & $\mathrm{~V}_{\mathrm{R}} \mathrm{HZ} \mathrm{R}_{\mathrm{R}+\mathrm{CU}}$ & 4313 & 727 & SD & 1204 & 5156 \\
\hline \multicolumn{12}{|c|}{ Time horizon $=85$ years } \\
\hline No vacc. & 3092 & 798 & - & - & - & No vacc. & 3724 & 1059 & - & - & - \\
\hline$V_{R}$ & 2962 & 759 & Cost-saving & 717 & 1695 & $V_{R}$ & 3704 & 1072 & 1517 & -174 & -497 \\
\hline$V_{R} H Z_{R}$ & 3984 & 634 & 8170 & 1570 & 5674 & $V_{R} H Z_{R}$ & 4572 & 874 & 4375 & 1934 & 6571 \\
\hline$H Z_{R}$ & 4119 & 676 & SD & 805 & 3859 & $H Z_{R}$ & 4627 & 878 & SD & 1824 & 6367 \\
\hline $\mathrm{V}_{\mathrm{R}} \mathrm{HZ} \mathrm{Z}_{\mathrm{R}+\mathrm{CU}}$ & 4264 & 600 & 8266 & 1799 & 6752 & $\mathrm{~V}_{\mathrm{R}} \mathrm{HZ} \mathrm{Z}_{\mathrm{R}+\mathrm{CU}}$ & 4842 & 834 & 6829 & 2257 & 7881 \\
\hline$H Z_{R+C U}$ & 4402 & 643 & SD & 1020 & 4904 & $\mathrm{HZ} \mathrm{Z}_{\mathrm{R}} \mathrm{CU}$ & 4898 & 839 & SD & 2128 & 7632 \\
\hline
\end{tabular}

All outcomes are reported with a $3 \%$ discount rate for both benefits and costs

${ }^{a}$ Accounting for cost of disease and cost of policy, in million EUR

${ }^{\mathrm{b}}$ In thousands

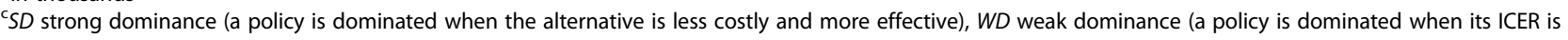
larger than that of a policy with higher effectiveness). The ICER is measured in EUR/QALY gained

${ }^{d}$ Based on the marginal productivity of the national health system ( $t=15,000$ EUR) and calculated with respect to no vaccination, in million EUR

'Based on the consumers' willingness to pay ( $t=40,000$ EUR) and calculated with respect to no vaccination, in million EUR

$\mathrm{V}_{\mathrm{R}} \mathrm{HZ}_{\mathrm{R}+\mathrm{CU}}$ to be the most cost-effective for all models and time horizons, except for $\mathrm{TH}=50$ under model $\mathrm{TI}$, where the policy $H Z_{R+C U}$ was the most cost-effective (Additional file 1: Table S5).

Under the PSA, we found that, for a CE threshold of $15,000 \mathrm{EUR}, \mathrm{V}_{\mathrm{R}}$ consistently underperforms compared to the other strategies, irrespective of the model used and the assumed discount rate. Its worst performance, with the NMB decreasing as the CE threshold increases, is expected under model PI with discount rate equal to $3 \%$, because of the higher (negative) impact of varicella vaccination on $\mathrm{HZ}$ epidemiology (Fig. 3h). On the contrary, under model TI, $V_{R}$ always generates a strictly positive NMB, but it is always dominated by the other strategies, except for $\mathrm{CE}$ thresholds lower than 8000 EUR (Fig. 3g).

Under both models, the combination of the two vaccinations, $V_{R} H Z_{R}$ and $V_{R} H Z_{R+C U}$, maximises the NMBs when considering undiscounted outcomes (Fig. 3a and b), with the latter strategy being the most cost-effective above a CE threshold of 8000 EUR (Fig. 3e and f). However, when assuming a $3 \%$ discount rate, we find that, under model PI, the reductions in HZ incidence in the long term are not enough to counterbalance the short-term increase in $\mathrm{HZ}$ infections induced by varicella vaccination. Hence, the resulting NMB distribution of the combined programmes is quite similar to those generated by $\mathrm{HZ}_{\mathrm{R}}$ and $\mathrm{HZ} Z_{\mathrm{R}+\mathrm{CU}}$ (Fig. $3 \mathrm{~d}$ and $\mathrm{h}$ ). In particular, increasing the $C E$ threshold, the strategy $V_{R} H Z_{R}+C U$ converges to a probability of about $60 \%$ of being the most cost-effective, while the strategy $\mathrm{HZ}_{\mathrm{R}+\mathrm{CU}}$ converges to about $40 \%$ (Additional file 1: Figure S14D). This result shows that the introduction of an $\mathrm{HZ}$ catch-up programme for those aged $66-75$ is always beneficial, as it usually produces an increase in the estimated NMB.

Interestingly, we found that the input parameters for the epidemiological model, rather than those for the economic model, represented the most influential source 


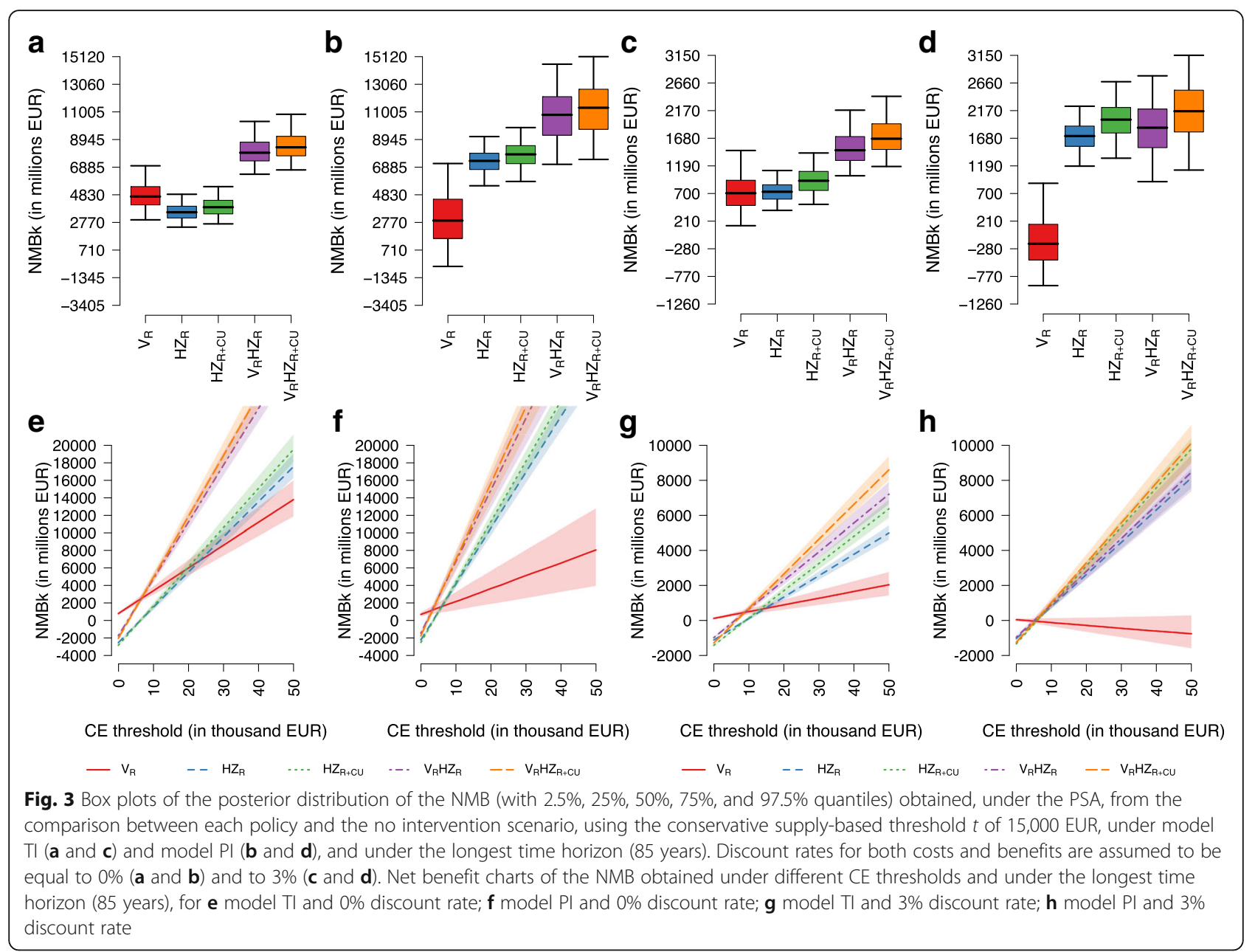

of uncertainty on the posterior distribution of the NMB (Additional file 1: Figure S26 and Table S6).

Remarkably, the obtained results are quite robust to variations in the varicella vaccination coverage levels, although upwards or downwards variations in the coverage generate, respectively, a decrease or an increase in the estimated NMBs (Additional file 1: Figures S15 and S16). When we assume a weaker boosting effect, i.e. a low reduction of the HZ risk due to VZV re-exposure, the distribution of the NMBs for the single $\mathrm{HZ}$ policies shifts downwards, and the combined policies $\mathrm{V}_{\mathrm{R}} \mathrm{HZ} \mathrm{R}_{\mathrm{R}+\mathrm{CU}}$ and $\mathrm{V}_{\mathrm{R}} \mathrm{HZ}_{\mathrm{R}}$ outperform the single ones (Additional file 1: Figure S17). Conversely, by considering a stronger role of boosting, both combined policies $\left(\mathrm{V}_{\mathrm{R}} \mathrm{HZ}_{\mathrm{R}}\right.$ and $\left.\mathrm{V}_{\mathrm{R}} \mathrm{HZ}+\mathrm{CU}\right)$ underperform compared to those solely based on $\mathrm{HZ}$ vaccination $\left(\mathrm{HZ}_{\mathrm{R}}\right.$ and $\left.\mathrm{HZ}_{\mathrm{R}+\mathrm{CU}}\right)$, while $\mathrm{V}_{\mathrm{R}}$ never proves cost-effective, even in the long term (Additional file 1: Figure S18). Considering the uncertainty in future demographic changes, scenarios assuming a higher future birth rate result in a higher varicella and a lower $\mathrm{HZ}$ incidence. However, under a high birth rate scenario, the population would also increase, leading to a higher number of $\mathrm{HZ}$ cases, despite the drop in the incidence. Nevertheless, in the long term, the results of the cost-effectiveness analysis are robust to the uncertainty in the demography, with the combined strategies generating the highest NMBs under both the low and the high birth rate scenarios (Additional file 1: Figures S23 and S24).

\section{Discussion \\ Principal findings}

The results of this study suggest that the recent introduction of the combined varicella and $\mathrm{HZ}$ vaccination programmes in Italy is expected to produce a significant reduction of the disease burden caused by VZV infection and reactivation in the long term. The new policy appears to be economically acceptable from a public health payer perspective under different model assumptions on the mechanism of exogenous boosting. In the base case analysis, under the progressive immunity (PI) model, we found that the combined programme would annually prevent, with respect to no vaccination, an average of 435,000 undiscounted cases of varicella, more than 77,000 cases of $\mathrm{HZ}$, and $81 \mathrm{HZ}$-related deaths per year 
at a cost of 4375 EUR per QALY gained. Instead, under the temporary immunity (TI) model, we would predict a reduction of almost 437,000 varicella cases, 59,000 HZ cases, and 45 HZ-related deaths per year at a cost of 8170 EUR per QALY gained. From our modelling results, the projected impact would be further improved by adding an $\mathrm{HZ}$ catch-up campaign targeting persons 66-75 years old to the implemented national programme. The inclusion of this targeted immunisation activity could prevent on average 3542 additional cases of $\mathrm{HZ}$ and 6 deaths per year under model PI, or 3079 $\mathrm{HZ}$ cases and 5 deaths under model TI, always maximising the estimated NMB of the strategy. The NMBs are mostly sensitive to the uncertainty on epidemiological parameters, and the ICER values strongly depend on parameters related to $\mathrm{HZ}$ and $\mathrm{PHN}$, such as the outpatient and inpatient costs and the corresponding QALY loss (Additional file 1: Figure S25). Overall results appeared robust to changes in model parameters and to different assumptions on exogenous boosting. However, when considering a post-vaccination time horizon of 25 years, varicella vaccination produced an increase in $\mathrm{HZ}$ incidence, the magnitude of which depended on whether we assumed a progressive or a temporary immunity model.

Individual strategies were also evaluated, and model results showed that, whereas $\mathrm{HZ}$ vaccination is expected to cost-effectively reduce the burden of $\mathrm{HZ}$ disease, varicella vaccination would negatively impact the overall burden of VZV in the short and the medium term. Hence, the introduction of this strategy on its own would not be considered cost-effective from the health care payer perspective.

\section{Strengths and limitations}

The obtained results are relevant as they thoroughly evaluate the impact of these newly introduced vaccination programmes, taking into consideration all potential direct and indirect, both positive and negative, effects of the vaccines in Italy, exploring the current uncertainty on the mechanism, either temporary or progressive, underlying exogenous boosting. This latter aspect differentiates this study from previous work, where the cost-effectiveness was evaluated either considering no exogenous boosting or assuming a TI mechanism. In the former case, findings showed that varicella vaccination was always cost-effective (under the health payer perspective) or even cost-saving (under the societal perspective) [27, 29], while in the latter case, it was generally not cost-effective [30, 31] (except for France [47]), except when considering the long term [29, 48]. Moreover, the model also improves on the previous analyses in the way demographic processes are accommodated. Indeed, the model explicitly considers realistic changes in the Italian population age structure over time to take into account temporal trends in VZV epidemiology that are not directly ascribable to the immunisation programme, but rather to the ageing of the population and to the reduction in the expected number of susceptible children. A similar approach was also recently considered for modelling varicella and HZ epidemiology in Germany [49]. In their study, the authors evaluated the effect of population ageing and migration flows on the epidemiology of the two diseases, along with vaccination policies, but they did not assess the cost-effectiveness and economic acceptability of the programmes.

Our work is based on the underlying assumption of long-lasting protection induced by the $\mathrm{HZ}$ vaccine, which might appear to contrast with recent evidence of declining effectiveness (from 60 to $70 \%$ in the first years to 30 to $40 \%$ in the eighth year) [50]. Although we acknowledge that this can be considered a limitation of our work, we believe that our assumption of a constant vaccine efficacy of $50 \%$ can be seen as an average level of protection throughout the observation period. To address the issue of waning vaccine-induced immunity, a second dose of vaccine has been suggested [51]. We took into consideration this possibility by doubling the price of the considered live attenuated $\mathrm{HZ}$ vaccine to mimic a two-dose $\mathrm{HZ}$ vaccination policy. We found that $\mathrm{HZ}$ vaccination would still be cost-effective in the long run, even under the lower threshold of the ICER. Clearly, more work is needed in this direction, also in the light of the fact that some countries are currently considering replacing the live attenuated $\mathrm{HZ}$ vaccine with a new recombinant one [52, 53]. This new vaccine has recently been licenced by the US Food and Drug Administration and recommended for healthy adults aged 50 and above to prevent shingles and related complications [54]. Although its reported high efficacy is expected to enhance the cost-effectiveness of the $\mathrm{HZ}$ vaccination, both alone and in combination with the varicella vaccine [55], the two-dose schedule and the high price might counterbalance the positive effects.

Finally, our model assumed that the varicella vaccination was first administered to all newborn babies in Italy beginning in 2017, despite the vaccination having gradually been available in some regions since 2003. Considering that the coverage in these regions has reached moderate or high levels in recent years [56, 57], our results might underestimate the impact of varicella vaccination on VZV circulation in Italy and consequently overestimate varicella and $\mathrm{HZ}$ incidence, mostly in the short term [4].

\section{Implications for policy makers}

Our findings are especially relevant when considering the very recent changes in the Italian National Immunisation Plan (NIP) 2017-2019. The NIP 2017-2019 has introduced recommendations for six new additional vaccinations, which will be administered free of charge: four vaccinations targeted to infants and children (i.e. vaccines 
against rotavirus, varicella, group $\mathrm{B}$ meningococcal disease, and human papillomavirus for boys), and two to the elderly (i.e. vaccines against pneumococcal disease and herpes zoster). These recommendations have sparked a lively and heated debate on the appropriateness of the new childhood programmes and on the fact that varicella is one of the ten vaccines (hexavalent, plus measles, mumps, rubella, and varicella) that have been introduced as compulsory for school attendance (only starting from those born in 2017). So far, Italy has shown high regional heterogeneity regarding immunisation schedules and outcomes; thus, a new structured national plan will promise a better harmonisation in the vaccine offer and uptake. At the same time, the NIP requirements for school entry should ensure the achievement and maintenance of the high vaccination coverage rates that are necessary for the desired herd immunity effects.

\section{Conclusions}

Our study has shown that the newly introduced combined varicella and $\mathrm{HZ}$ vaccination strategy in Italy is expected to be effective and cost-effective in reducing the burden of disease and the loss of quality of life. Moreover, the programme is expected to counterbalance the increasing trend in $\mathrm{HZ}$ incidence that is estimated in the absence of any vaccination programme and is thus purely due to demographic change. In particular, under the more realistic assumption of PI for the exogenous boosting, this decline would amount to around 435,000 undiscounted cases of varicella and more than 77,000 cases of HZ (and $81 \mathrm{HZ}$-related deaths) per year at a cost of 4375 EUR per QALY gained. We also found that an additional catch-up campaign for $\mathrm{HZ}$ vaccination targeting people aged 66-75 would further increase the benefits of the combined programme, leading to an additional reduction of 3542 cases of $\mathrm{HZ}$ and $6 \mathrm{HZ}$-related deaths per year, at a cost of 6829 EUR per QALY gained.

Our work shows the importance of using models with non-stationary populations, in particular accounting for changing demography, when assessing the impact of vaccination policies on the epidemiology of those infectious diseases that are highly dependent on people mixing. This can surely provide a more thorough understanding of the expected outcomes and therefore help policy makers to design effective preventive strategies.

\section{Additional file}

Additional file 1: Supplementary methods and results. (DOCX 6670 kb)

\section{Abbreviations}

CE: Cost-effectiveness; Cl: Credible interval; GP: General practitioner; HZ: Herpes zoster; IBM: Individual-based model; ICER: Incremental costeffectiveness ratio; MCMC: Monte Carlo Markov chain; NIP: National Immunisation Plan; NMB: Net monetary benefit; PHN: Post-herpetic neuralgia;
PI: Progressive immunity; PSA: Probabilistic sensitivity analysis; QALY: Qualityadjusted life year; TH: Time horizon; TI: Temporary immunity; VZV: Varicella zoster virus

\section{Acknowledgements}

We are grateful to Dr. Maria Gramegna and Alessandra Piatti from Regione Lombardia for providing us with the Hospital Discharge Register data on varicella and $\mathrm{HZ}$ for the Lombardy region. We also thank Prof. Giovanni Fattore and Carlo Federici from Bocconi University for the helpful discussions and for providing feedback on our work. We thank the reviewers for their very useful comments and suggestions.

\section{Funding}

The research leading to these results has received funding from the European Research Council (ERC) under the European Union's Seventh Framework Programme (FP7/2007-2013) and ERC Grant agreement number 283955 (DECIDE). The funder had no role in the design of the study, collection, analysis, or interpretation of data, or in writing the manuscript.

Availability of data and materials

All epidemiological and economic data used for the analysis are reported in Additional file 1 and in Tables 1 and 2 of the paper.

\section{Authors' contributions}

AM, MT, and PP conceived the project. MT collected all data on economic parameters. VM, PP, and SM designed the computer model and carried out the computer simulations and analysis. EDF and AM designed the economic model. EDF performed the cost-effectiveness analysis. CR provided epidemiological expertise. AM, VM, and EDF drafted the manuscript. All authors commented on the drafts and contributed to the final version. AM is the guarantor of the study. All authors read and approved the final manuscript.

Ethics approval and consent to participate Not applicable.

\section{Competing interests}

PP has received personal fees from Merck \& Co. Inc. for consultancy activity outside of the submitted work. All other authors declare that they have no competing interests.

\section{Publisher's Note}

Springer Nature remains neutral with regard to jurisdictional claims in published maps and institutional affiliations.

\section{Author details}

${ }^{1}$ Department of Social and Political Sciences, Bocconi University, Milano, Italy. ${ }^{2}$ Carlo F. Dondena Centre for Research on Social Dynamics and Public Policy, Bocconi University, Milano, Italy. ${ }^{3}$ Center for Information and Communication Technology, Bruno Kessler Foundation, Trento, Italy. ${ }^{4}$ Department of Hygiene and Preventive Medicine, ATS, Bergamo, Italy. ${ }^{5}$ Department of Infectious

Disease, Istituto Superiore di Sanità, Roma, Italy.

Received: 12 January 2018 Accepted: 5 June 2018

Published online: 17 July 2018

\section{References}

1. Gauthier A, Breuer J, Carrington D, Martin M, Rémy V. Epidemiology and cost of herpes zoster and post-herpetic neuralgia in the United Kingdom. Epidemiol Infect. 2008;137:38-47.

2. Gialloreti LE, Merito M, Pezzotti P, Naldi L, Gatti A, Beillat M, et al. Epidemiology and economic burden of herpes zoster and post-herpetic neuralgia in Italy: a retrospective, population-based study. BMC Infect Dis. 2010;10:230-11.

3. Goldman GS, King PG. Review of the United States universal varicella vaccination program: herpes zoster incidence rates, cost-effectiveness, and vaccine efficacy based primarily on the Antelope Valley Varicella Active Surveillance Project data. Vaccine. 2013;31:1680-94.

4. Bechini A, Boccalini S, Baldo V, Castiglia P, Gallo T, Giuffrida S, et al. Impact of universal vaccination against varicella in Italy. Hum Vaccin Immunother. 2015;11:63-71. 
5. Signorelli C, Guerra R, Siliquini R, Ricciardi W. Italy's response to vaccine hesitancy: an innovative and cost effective National Immunization Plan based on scientific evidence. Vaccine. 2017;35:4057-9.

6. Brisson M, Edmunds WJ, Gay NJ. Varicella vaccination: impact of vaccine efficacy on the epidemiology of VZV. J Med Virol. 2003;70:531-7.

7. Hope-Simpson RE. The nature of herpes zoster: a long-term study and a new hypothesis. Proc R Soc Med. 1965;58:9-20.

8. Jumaan AO, Yu O, Jackson LA, Bohlke K, Galil K, Seward JF. Incidence of herpes zoster, before and after varicella-vaccination-associated decreases in the incidence of varicella, 1992-2002. J Infect Dis. 2005;191:2002-7.

9. Reynolds MA, Chaves SS, Harpaz R, Lopez AS, Seward JF. The impact of the varicella vaccination program on herpes zoster epidemiology in the United States: a review. J Infect Dis. 2008;197:S224-7.

10. Kawai K, Gebremeskel BG, Acosta CJ. Systematic review of incidence and complications of herpes zoster: towards a global perspective. BMJ Open. 2014;4:e004833.

11. Marziano V, Poletti P, Guzzetta G, Ajelli M, Manfredi P, Merler S. The impact of demographic changes on the epidemiology of herpes zoster: Spain as a case study. Proc R Soc B. 2015;282:20142509.

12. Mitka M. FDA approves shingles vaccine: herpes zoster vaccine targets older adults. J Am Med Assoc. 2006;296:157-8.

13. Oxman MN, Levin MJ, Johnson GR, Schmader KE, Straus SE, Gelb LD, et al. A vaccine to prevent herpes zoster and postherpetic neuralgia in older adults. N Engl J Med. 2009;352:2271-84.

14. Karhunen M, Leino T, Salo H, Davidkin I, Kilpi T, Auranen K. Modelling the impact of varicella vaccination on varicella and zoster. Epidemiol Infect. 2010;138:469-81.

15. Poletti P, Melegaro A, Ajelli M, Del Fava E, Guzzetta G, Faustini L, et al. Perspectives on the impact of varicella immunization on herpes zoster. A modelbased evaluation from three European countries. PLoS One. 2013;8:e60732.

16. Brisson M, Melkonyan G, Drolet M, De Serres $G$, Thibeault $R$, De Wals $P$. Modeling the impact of one- and two-dose varicella vaccination on the epidemiology of varicella and zoster. Vaccine. 2010;28:3385-97.

17. van Hoek AJ, Melegaro A, Zagheni E, Edmunds WJ, Gay NJ. Modelling the impact of a combined varicella and zoster vaccination programme on the epidemiology of varicella zoster virus in England. Vaccine. 2011;29:2411-20.

18. Guzzetta G, Poletti P, Del Fava E, Ajelli M, Scalia Tomba G, Merler S, et al. Hope-Simpson's progressive immunity hypothesis as a possible explanation for herpes zoster incidence data. Am J Epidemiol. 2013;177:1134-42.

19. Guzzetta G, Poletti P, Merler S, Manfredi P. The epidemiology of herpes zoster after varicella immunization under different biological hypotheses: perspectives from mathematical modeling. Am J Epidemiol. 2016;183:765-73.

20. Terada K, Kawano S, Yoshihiro K, Morita T. Proliferative response to varicellazoster virus is inversely related to development of high levels of varicellazoster virus specific lgG antibodies. Scand J Infect Dis. 2009;25:775-8.

21. Vossen MTM, Gent M-R, Weel JFL, de Jong MD, van Lier RAW, Kuijpers TW. Development of virus-specific CD4+ T cells on reexposure to varicella-zoster virus. J Infect Dis. 2004;190:72-82.

22. Ogunjimi B, Smits E, Hens N, Hens A, Lenders $K$, leven $M$, et al. Exploring the impact of exposure to primary varicella in children on varicella-zoster virus immunity of parents. Viral Immunol. 2011;24:151-7.

23. Thomas SL, Wheeler JG, Hall AJ. Contacts with varicella or with children and protection against herpes zoster in adults: a case-control study. Lancet. 2002;360:678-82.

24. Brisson M, Gay NJ, Edmunds WJ, Andrews N. Exposure to varicella boosts immunity to herpes-zoster: implications for mass vaccination against chickenpox. Vaccine. 2002;20:2500-7.

25. Gaillat J, Gajdos V, Launay O, Malvy D, Demoures B, Lewden L, et al. Does monastic life predispose to the risk of Saint Anthony's fire (herpes zoster)? Clin Infect Dis. 2011:53:405-10.

26. Marangi L, Mirinaviciute G, Flem E, Scalia Tomba G, Guzzetta G, Freiesleben de Blasio B et al. The natural history of varicella zoster virus infection in Norway: Further insights on exogenous boosting and progressive immunity to herpes zoster. PLoS ONE. 2017; 12: e0176845-17

27. Damm O, Ultsch B, Horn J, Mikolajczyk RT, Greiner W, Wichmann O. Systematic review of models assessing the economic value of routine varicella and herpes zoster vaccination in high-income countries. BMC Public Health. 2015;15:222.

28. van Lier A, Lugnér A, Opstelten W, Jochemsen P, Wallinga J, Schellevis F, et al. Distribution of health effects and cost-effectiveness of varicella vaccination are shaped by the impact on herpes zoster. EBioMedicine. 2015;2:1494-9.
29. Bilcke J, van Hoek AJ, Beutels P. Childhood varicella-zoster virus vaccination in Belgium: cost-effective only in the long run or without exogenous boosting? Hum Vaccin Immunother. 2014;9:812-22.

30. Brisson M, Edmunds WJ. The cost-effectiveness of varicella vaccination in Canada. Vaccine. 2002;20:1113-25.

31. Brisson M, Edmunds WJ. Varicella vaccination in England and Wales: costutility analysis. Arch Dis Child. 2003;88:862-9.

32. Human Mortality Database University of California, Berkeley (USA), and Max Planck Institute for Demographic Research (Germany) http://www.mortality.org. Accessed 3 May 2018.

33. Database of the Italian Institute of Statistics. Italian National Institute of Statistics (ISTAT), Rome, Italy. http://demo.istat.it/. Accessed 3 May 2018.

34. Merler S, Ajelli M. Deciphering the relative weights of demographic transition and vaccination in the decrease of measles incidence in Italy. Proc R Soc B. 2014:281:20132676

35. Trentini F, Poletti P, Merler S, Melegaro A. Measles immunity gaps and the progress towards elimination: a multi-country modelling analysis. Lancet Infect Dis. 2017;17:1089-97.

36. Johnson RW. Zoster-associated pain: what is known, who is at risk and how can it be managed? Herpes. 2007;14((Suppl 2)):30-4.

37. Seward JF, Zhang JX, Maupin TJ, Mascola L, Jumaan AO. Contagiousness of varicella in vaccinated cases: a household contact study. J Am Med Assoc. 2004:292:704-8.

38. Civen R, Chaves SS, Jumaan AO, Wu H, Mascola L, Gargiullo PM, et al. The incidence and clinical characteristics of herpes zoster among children and adolescents after implementation of varicella vaccination. Pediatr Infect Dis J. 2009:28:954-9.

39. Ministero della Salute. Piano Nazionale Prevenzione Vaccinale. Rome: Gazzetta Ufficiale; 2017.

40. Vázquez M, LaRussa PS, Gershon AA, Steinberg SP, Freudigman K, Shapiro ED. The effectiveness of the varicella vaccine in clinical practice. N Engl J Med. 2001;344:955-60

41. Gabutti G, Penna C, Rossi M, Salmaso S, Rota MC, Bella A, et al. The seroepidemiology of varicella in Italy. Epidemiol Infect. 2001;126:433-40.

42. Fattore $\mathrm{G}$. Proposta di linee guida per la valutazione economica degli interventi sanitari in Italia. Pharmacoeconomics Ital Res Articles. 2009;11:83-93.

43. Woods B, Revill P, Sculpher M, Claxton K. Country-level cost-effectiveness thresholds: initial estimates and the need for further research. Value Health. 2016;19:929-35.

44. Baio G, Dawid AP. Probabilistic sensitivity analysis in health economics. Stat Methods Med Res. 2011;24:615-34

45. Briggs $\mathrm{AH}$. A Bayesian approach to stochastic cost-effectiveness analysis. Health Econ. 1999;8:257-61.

46. Stinnett AA, Mullahy J. Net health benefits: a new framework for the analysis of uncertainty in cost-effectiveness analysis. Med Dec Making. 1998;18:202-12.

47. Littlewood KJ, Ouwens MJNM, Sauboin C, Tehard B, Alain S, Denis F. Costeffectiveness of routine varicella vaccination using the measles, mumps, rubella and varicella vaccine in France: an economic analysis based on a dynamic transmission model for varicella and herpes zoster. Clin Ther. 2015;37:830-7.

48. van Hoek AJ, Melegaro A, Gay NJ, Bilcke J, Edmunds WJ. The cost-effectiveness of varicella and combined varicella and herpes zoster vaccination programmes in the United Kingdom. Vaccine. 2012; 30:1225-34.

49. Horn J, Damm O, Greiner W, Hengel H, Kretzschmar ME, Siedler A, et al. Influence of demographic changes on the impact of vaccination against varicella and herpes zoster in Germany - a mathematical modelling study. BMC Med. 2018;16:1-9.

50. Morrison VA, Johnson GR, Schmader KE, Levin MJ, Zhang JH, Looney DJ, et al. Long-term persistence of zoster vaccine efficacy. Clin Infect Dis. 2015:60:900-9.

51. Tseng H-F, Harpaz R, Luo Y, Hales CM, Sy LS, Tartof SY, et al. Declining effectiveness of herpes zoster vaccine in adults aged $\geq 60$ years. J Infect Dis. 2016;213:1872-5.

52. Lal H, Cunningham AL, Godeaux O, Chlibek R, Díez-Domingo J, Hwang S-J, et al. Efficacy of an adjuvanted herpes zoster subunit vaccine in older adults. N Engl J Med. 2015:372:2087-96.

53. Cunningham AL, Lal H, Kovac M, Chlibek R, Hwang S-J, Díez-Domingo J, et al. Efficacy of the herpes zoster subunit vaccine in adults 70 years of age or older. N Engl J Med. 2016;375:1019-32.

54. Span P. No excuses, people: get thenew shingles vaccine. New York City: The New York Times; 2017. https://nyti.ms/2ho3vmE. Accessed 3 May 2018 
55. Marchetti S, Guzzetta G, Flem E, Mirinaviciute G, Scalia Tomba G, Manfredi P. Modeling the impact of combined vaccination programs against varicella and herpes zoster in Norway. Vaccine 2018; 36: 1116-125.

56. Trucchi C, Gabutti G, Rota MC, Bella A. Burden of varicella in Italy, 2001-2010: analysis of data from multiple sources and assessment of universal vaccination impact in three pilot regions. J Med Microbiol. 2015;64:1387-94.

57. Signorelli C, Odone A, Cella P, lannazzo S, D'Ancona F, Guerra R. Infant immunization coverage in Italy (2000-2015). Ann Ist Super Sanità. 2017; 53:231-7.

58. Vázquez M, Shapiro ED. Varicella vaccine and infection with varicella-zoster virus. N Engl J Med. 2005;352:439-40.

59. Thiry N, Beutels P, Van Damme P, Van Doorslaer E. Economic evaluations of varicella vaccination programmes. PharmacoEconomics. 2003;21:13-38.

60. Coudeville L, Brunot A, Szucs TD, Dervaux B. The economic value of childhood varicella vaccination in France and Germany. Value Health. 2005; 8:209-22.

61. Kind P, Hardman G, Macran S. UK population norms for EQ-5D. Centre for Health Economics Discussion Paper Series. Report No. 172. York: University of York; 1999. p. 1-98.

62. Bala MV, Wood LL, Zarkin GA, Norton EC, Gafni A, O'Brien B. Valuing outcomes in health care: a comparison of willingness to pay and qualityadjusted life-years. J Clin Epidemiol. 1998;51:667-76.

63. Chaves SS, Zhang J, Civen R, Watson BM, Carbajal T, Perella D, et al. Varicella disease among vaccinated persons: clinical and epidemiological characteristics, 1997-2005. J Infect Dis. 2008;197(Suppl 2):S127-31.

64. Fornaro P, Gandini F, Marin M, Pedrazzi C, Piccoli P, Tognetti D, et al. Epidemiology and cost analysis of varicella in Italy: results of a sentine study in the pediatric practice. Pediatr Infect Dis J. 1999;18:414-9.

65. Zotti CM, Maggiorotto G, Migliardi A. I costi della varicella. Ann Ig 2002;14:29-33.

66. Giaquinto C, Sturkenboom M, Mannino S, Arpinelli F, Nicolosi A, Cantarutti L. Epidemiologia ed esiti della varicella in Italia: risultati di uno studio prospettico sui bambini (0-14 anni) seguiti dai pediatri di libera scelta (Studio Pedianet). Ann Ig. 2002;14:21-7.

\section{Ready to submit your research? Choose BMC and benefit from:}

- fast, convenient online submission

- thorough peer review by experienced researchers in your field

- rapid publication on acceptance

- support for research data, including large and complex data types

- gold Open Access which fosters wider collaboration and increased citations - maximum visibility for your research: over $100 \mathrm{M}$ website views per year

At BMC, research is always in progress.

Learn more biomedcentral.com/submissions 